\title{
Diagnosis and Management of Castleman Disease
}

\author{
Presented by Jeremy S. Abramson, MD, MMSc
}

\begin{abstract}
Castleman disease is a heterogeneous nonmalignant lymphoproliferative disorder. Major distinctions include unicentric versus multicentric presentation; hyaline vascular, plasmacytic, or mixed pathology; and HHV8-associated (typically HIV-positive) versus idiopathic disease. At the NCCN 2019 Annual Congress: Hematologic Malignancies, Dr. Jeremy S. Abramson stated that rituximab is preferred as initial therapy for HHV8-positive disease, and chemotherapy can be added for patients with fulminant disease (antiretrovirals should always be used as well for those who are HIV-positive). Siltuximab is the preferred frontline therapy for idiopathic disease.
\end{abstract}

J Natl Compr Canc Netw 2019;17(11.5):1417-1419 doi: $10.6004 /$ jnccn.2019.5037

Castleman disease is a nonmalignant lymphoproliferative disorder that, although uncommon, is a relatively complex disease to diagnose and manage, according to Jeremy S. Abramson, MD, MMSc, Associate Professor of Medicine, Harvard Medical School, and Director of the Lymphoma Center, Massachusetts General Hospital Cancer Center. "Castleman disease is a heterogeneous group of diseases under one heading," he explained at the NCCN 2019 Annual Congress: Hematologic Malignancies. "It is something that warrants thinking about, particularly given that we have moved effectively from the era of chemotherapy into the era of novel targeted strategies in Castleman disease as we have in so many other lymphoproliferative diseases."

\section{Classification of Castleman Disease}

Castleman disease is characterized by 2 pathologic subtypes. In hyaline vascular tumors, mantle zones surrounding atrophic germinal centers are expanded with concentric rings of small lymphocytes, thereby giving them an "onion skin" appearance. Increased vascularity is observed and blood vessels may be seen penetrating germinal centers. The plasmacytic (and occasionally plasmablastic) variant is a more polymorphous inflammatory infiltrate with a high proportion of polyclonal plasma cells. "And to make things a little more complicated, we can see an overlap in features, which is called "mixed histology with features of both hyaline vascular and plasmacytic'," he said.

Clinically, Castleman disease is classified as either unicentric (UCD) or multicentric (MCD), and the latter presentation occurs in several different varieties: human herpesvirus-8 (HHV-8)-associated disease occurs mostly in the setting of HIV or other immunocompromised states, whereas HHV8-negative disease is referred to as idiopathic MCD (iMCD), and is further classified as iMCD-TAFRO (thrombocytopenia, anasarca, fevers, reticulin myelofibrosis, organomegaly) or iMCD-NOS (not otherwise specified). The least common subtype of MCD is POEMS-associated. In addition, Dr. Abramson noted that iMCD-TAFRO is a marked inflammatory syndrome, thus these patients are often extremely ill, usually with high fevers and may have renal failure, but have normal immunoglobulin levels. iMCD-NOS tends to have a "gentler" clinical presentation.

\section{Diagnosing Castleman Disease}

According to Dr. Abramson, diagnosing Castleman disease can be complicated. "A lot of the effort falls on our hematopathology colleagues, of course, but it also falls on us," he said. "It's important to recognize the clinical features of Castleman, which can be missed. So if you are concerned about this diagnosis, make sure to interface with the diagnosing pathologist."

When conducting a diagnostic workup, HIV status must be evaluated, as well as baseline serum inflammatory markers. "We usually check IL-6 at baseline because that can be a biomarker for the disease," he noted. Another critical factor to note is the high degree of overlap between Castleman disease in the setting of HIV and Kaposi's sarcoma (KS). "The KS herpesvirus is, in fact, HHV-8, and it is estimated that more than half of patients presenting with a new diagnosis of HIV-associated Castleman disease can also have concomitant KS," he explained. "So please evaluate these patients carefully, both cutaneously and considering symptoms of visceral involvement." 
Dr. Abramson also discussed the importance of recognizing mimics of Castleman disease. Lymphomas, including Hodgkin and angioimmunoblastic T-cell lymphoma, are also known for polymorphous infiltrate and hypervascularity, making them prime culprits for mimicking the disease. Other mimics include autoimmune diseases (particularly IgG4-related disease, but also lupus and rheumatoid arthritis), as well as inflammatory infectious responses to HIV, Epstein-Barr virus, and syphilis.

\section{Treating UCD}

UCD is almost always hyaline vascular subtype, is usually HIV-negative and HHV8-negative, and commonly presents in younger adults (aged 30-40 years), with a slight predominance in women. Common sites of presentation include the chest (30\%), neck (23\%), abdomen (20\%), and less commonly, the retroperitoneum. Furthermore, UCD usually presents as localized, asymptomatic adenopathy, and is commonly diagnosed through incidental discovery on radiographic imaging performed for a different reason. "Now that we have patients getting CT scans every time they have a stuffy nose, we are finding a lot more incidental adenopathy and masses than we might have otherwise," he joked.

The treatment of choice for UCD is surgical resection, which is associated with $>90 \%$ relapse-free survival. Unresectable disease can be treated with local radiation, or rituximab and/or steroids can be used to reduce the mass with the goal of converting it to be surgically resectable. Embolization has also been used with some success. If the mass does not shrink after these interventions, Dr. Abramson stressed the nonprogressive, benign nature of the disease and warned of the risk of overtreatment. "Usually I'll either radiate these patients if I can't surgically resect or I'll observe," he said. "I usually do not use systemic therapy for this disease when it is unicentric."

\section{Treating MCD}

HHV8-positive MCD is generally associated with an IL-6-mediated inflammatory syndrome, and is most commonly seen in the setting of HIV (but may rarely occur in other immunocompromised patients). It is most commonly plasmacytic or mixed histology, with a male predominance. Patients with MCD are often quite ill and typically present with fevers, diffuse lymphadenopathy, hepatosplenomegaly, elevated inflammatory markers, cytopenias, and hypoalbuminemia. Dr. Abramson also pointed out the increased risk of opportunistic infections in these patients, and stated that multidisciplinary care including infectious disease specialists is critical.

Prognosis for HHV8-positive MCD has improved dramatically over time due to highly active antiretroviral

CRITERIA FOR ACTIVE DISEASE ${ }^{\mathrm{a}}$
- Fever
- Increased serum C-reactive protein level > $20 \mathrm{mg} / \mathrm{L}$ in the
absence of any other etiology
- At least three of the following other MCD-related symptoms:
- Peripheral lymphadenopathy
- Enlarged spleen
- Edema
Pleural effusion
- Ascites
- Cough
- Nasal obstruction
- Xerostomia
Rash
- Central neurologic symptoms
Jaundice
- Autoimmune hemolytic anemia

Figure 1. Criteria for active disease in the NCCN Guidelines. Version 5.2019. To view the most recent version, visit NCCN.org.

therapy for HIV and modern biologic therapy for Castleman disease (most notably rituximab). Previously, the disease was treated primarily with chemotherapy, but this was poorly tolerated by patients with HIV and was associated with high rates of treatment-related morbidity and mortality. "Certainly, all patients with HIV should be started on antiretroviral therapy," he said. "But unlike some viral diseases with associated lymphomas, you will not see a regression of Castleman disease with antiretroviral therapy directed at HIV alone. So patients will need Castleman disease-directed therapy."

HHV8-associated MCD is virtually always symptomatic, but it is important to note that treatment is only indicated when active disease is present (ie, asymptomatic patients can be observed) (Figure 1).

\section{Rituximab as Initial Treatment in HHV8-Positive Disease}

Data guiding choice of therapy in HHV8-positive disease come from small trials, but rituximab has been established as first-line therapy in these patients. In the CastlemaB trial, $^{1}$ the first prospective study of rituximab in $\mathrm{HIV}$-associated MCD, 24 patients with relapsed/refractory, chemotherapy-dependent disease were given 4 weekly doses of rituximab at $375 \mathrm{mg} / \mathrm{m}^{2}$. "Remarkably, 22 of the 24 patients were able to become independent of chemotherapy," noted Dr. Abramson. Most patients responded clinically, and more than threequarters remained free from symptoms and progression 2 years after treatment.

In another retrospective analysis of 113 patients, 48 had previously received rituximab and 60 were previously diagnosed with KS. ${ }^{2}$ Patients treated with rituximab had a 5-year overall survival rate of $90 \%$ compared with $47 \%$ for those treated with chemotherapy alone. However, among 24 patients with prior 
KS who received rituximab, 8 experienced exacerbation of their KS and 1 new case of KS occurred. "This is a real phenomenon that we see in our clinic," he said. "Patients with concurrent HIV-associated KS and Castleman can have a flare of their KS while being treated with rituximab monotherapy." For these patients, alternate regimens containing rituximab and liposomal doxorubicin (in addition to antiretroviral therapy) should be considered in the front line. ${ }^{3}$

Chemotherapy options, such as etoposide, vinblastine, liposomal doxorubicin, or combination chemotherapy regimens remain available in the relapsed/ refractory setting. For patients with highly symptomatic or organ-threatening disease who need a more rapid response, combination regimens such as R-CHOP (rituximab/cyclophosphamide/doxorubicin/vincristine/ prednisone) should be considered.

\section{Siltuximab for iMCD}

For HHV8-negative disease (iMCD), "go down the siltuximab route for most patients," advised Dr. Abramson. A double-blind international trial that randomized patients with iMCD 2:1 to the IL-6 antibody siltuximab or placebo $^{4}$ showed a durable tumor and symptomatic response rate of $34 \%$ for siltuximab versus $0 \%$ for placebo, with a duration of response of $>1$ year. Patients receiving siltuximab also experienced significant improvements in durable symptom response compared with placebo ( $57 \%$ vs $19 \%$ ) and anemia ( $61 \%$ vs $0 \%$ ). "Interestingly, this benefit was observed exclusively in patients with the plasmacytic or mixed histologic variants; no patients with hyaline vascular pathology responded to this regimen," he said. "I would consider those patients high risk for not responding to siltuximab therapy."

Dr. Abramson also noted that siltuximab is not particularly effective in HHV8-associated MCD because the drug only binds to human IL- 6 and not viral IL-6, which is also elevated in HHV8-positive disease. ${ }^{5}$ Although the NCCN Clinical Practice Guidelines in Oncology (NCCN Guidelines) for B-Cell Lymphomas list rituximab and prednisone as a frontline treatment option, ${ }^{6}$ he maintained that siltuximab is the treatment of choice for iMCD. "[Rituximab and prednisone] is not my first choice because the data are really an extrapolation from HIV/HHV8-associated MCD," he said. "Currently the data predominantly support siltuximab as initial therapy for iMCD."

A thalidomide/cyclophosphamide/prednisone regimen is an additional option in patients with relapsed disease, ${ }^{7}$ and can also be considered in the frontline treatment of patients with hyaline vascular $\mathrm{MCD}$, he said.

Beyond chemotherapy for the treatment of highly refractory disease, data from case reports suggest that the use of certain targeted agents as monotherapy may be effective (sirolimus, anakinra, bortezomib, lenalidomide). However, Dr. Abramson added, these data are scarce.

Disclosures: Dr. Abramson has disclosed that he receives consulting fees from AbbVie, Inc., Bayer HealthCare, Celgene Corporation, EMD Serono, Genentech, Inc., Janssen Pharmaceutica Products, LP, Karyopharm Therapeutics, Kite Pharma, and Roche Laboratories, Inc.

Correspondence: Jeremy S. Abramson, MD, MMSc, Massachusetts General Hospital Cancer Center, 55 Fruit Street, Boston, MA 02114. Email: jabramson@mgh.harvard.edu

\section{References}

1. Gérard L, Bérezné A, Galicier L, et al. Prospective study of rituximab in chemotherapydependent human immunodeficiency virus associated multicentric Castleman's disease: ANRS 117 CastlemaB trial. J Clin Oncol 2007;25:3350-3356.

2. Gérard L, Michot JM, Burcheri S, et al. Rituximab decreases the risk of lymphoma in patients with HIV-associated multicentric Castleman disease. Blood 2012;119:2228-2233.

3. Uldrick TS, Polizzotto MN, Aleman K, et al. Rituximab plus liposomal doxorubicin in HIV-infected patients with KSHV-associated multicentric Castleman disease. Blood 2014;124:3544-3552.

4. van Rhee F, Wong RS, Munshi N, et al. Siltuximab for multicentric Castleman's disease: a randomised, double-blind, placebo-controlled trial. Lancet Oncol 2014;15:966-974.
5. Polizzotto MN, Uldrick TS, Wang $\mathrm{V}$, et al. Human and viral interleukin-6 and other cytokines in Kaposi sarcoma herpesvirus-associated multicentric Castleman disease. Blood 2013; 122:4189-4198.

6. Zelenetz AD, Gordon LI, Abramson JS, et al. NCCN Clinical Practice Guidelines in Oncology: B-Cell Lymphomas. Version 5.2019. To view the most recent version, visit NCCN.org. Accessed October 15 2019.

7. Zhang L, Zhao AL, Duan $\mathrm{MH}$, et al. Phase 2 study using oral thalidomide-cyclophosphamide-prednisone for idiopathic multicentric Castleman disease. Blood 2019;133:

1720-1728. 